\title{
Study of telemetry changes over time in children with a cochlear implant
}

\author{
Samir I. Asal ${ }^{a}$, Osama A. Sobhy ${ }^{a}$, Najla D. Massad ${ }^{b}$
}

Departments of atorhinolaryngology, ${ }^{\mathrm{b}}$ AudioVestibular Medicine, Audiology, Faculty of Medicine, Alexandria University, Alexandria Egypt

Correspondence to Najla D. Massad, MSc, Department of Audiology, Faculty of Medicine, Alexandria University, Alexandria, Egypt. Tel: +201224549955;

e-mail: najla-dafallah1317@hotmail.com

Received 7 July 2017

Accepted 10 September 2017

The Egyptian Journal of Otolaryngology 2018, 34:198-202

\begin{abstract}
Background
Cochlear implant $(\mathrm{Cl})$ is an electronic device that provides direct electrical stimulation to the auditory division of the eighth cranial nerve. The integrity of the internal $\mathrm{Cl}$ part after implantation can be assessed through objective measures, which are a widely used and valuable tool in the field of Cls. Impedance measurement and electrically evoked compound action potential (ECAP) are the most frequently used tests to facilitate programming of the implants especially in young children.

Aim

This study was carried out to compare ECAP thresholds and electrode impedance at the time of surgery, at the first stimulation session, and monthly for the next 2 months to assess whether a significant change take places with time.

Patients and methods

Fifteen deaf children implanted unilaterally with a MED-EL Sonata Implant System with an Opus 2 speech processor were included in this study. All patients received the implant if they fulfilled the Alexandria main hospital criteria for receiving Cls. The group studied included seven males and eight females; they ranged in age from 2 to 6 years. Two of the patients had received implantation in the left ear, whereas the rest of the patients had received a $\mathrm{Cl}$ in the right ear.

Results

Intraoperative impedance was the lowest among all postoperative readings in all electrodes. The highest value was that measured 1 month after surgery, after which impedance values continued to decrease significantly, but not to the intraoperative values. The ECAP threshold showed no significant $P$ values between the ECAP threshold measured in the intraoperative and the postsurgery follow-up period. Conclusion

The measured impedance showed significant changes in the form of increasing values postoperatively relative to the intraoperative time. The ECAP threshold did not change significantly intraoperatively and postoperatively, showing that intraoperative ECAP can be useful in mapping as it shows no changes.
\end{abstract}

\section{Keywords:}

electrically evoked compound action potential, impedance, telemetry

Egypt J Otolaryngol 34:198-202

(C) 2018 The Egyptian Journal of Otolaryngology

$1012-5574$

\section{Introduction}

Cochlear implantations are nowadays considered an optimal solution option in profoundly hearing impaired populations as it has a high successful rate when preceded by thorough preoperative evaluations and preparations.

In cochlear implant (CI) users, each CI electrode interacts with nearby neurons in a particular way and this quality of the interaction is also expected to be a relevant factor according to the position and surrounding of the electrode inside the cochlea, the spread of electrical current to the neurons, and the local neural survival pattern [1].

Three manufacturers provide CI systems in the US markets and have received FDA approval: Advanced Bionics (Los Angeles, California, USA), Cochlear
Americas (Centennial, Colorado, USA), and MEDEL (Innsbruck, Austria). All of the systems developed by these manufacturers include an internal implanted device and an external part, which is the speech processor [2].

The integrity of this CI can be assessed intraoperatively and followed postoperatively through objective measures. These objective measures are used widely and are nowadays considered a valuable tool in the field of CIs because intraoperatively, they are used as the first indicator of successful implant placement and after surgery, they are used to guide the individual fitting of

This is an open access journal, and articles are distributed under the terms of the Creative Commons Attribution-NonCommercial-ShareAlike 4.0 License, which allows others to remix, tweak, and build upon the work non-commercially, as long as appropriate credit is given and the new creations are licensed under the identical terms. 
stimulation parameters, especially in young children, and also to monitor implant integrity each time the patient is tested [3]. These tests can be performed easily because they are noninvasive and measurement can be repeated as needed [4].

Also, this telemetry system provides information on the functionality of the CI, the electrical current interaction between electrodes, the impedance and the functionality of each electrode [5].

The most objective electrophysiological tests (telemetry tests) that are currently available and used by most CI programmers include telemetric measurement of electrode impedance, recording of the electrically evoked compound action potential (ECAP), in the cochlear nerve observation of the stapedial reflex, and electrically evoked auditory brain stem response [4].

The electrode impedance is a method of measuring resistance encountered by electricity while passing through wires, electrodes, and biological tissue. It is calculated as the ratio of effective voltage applied to a particular circuit and the actual amount of electrical power intensity absorbed by the circuit. The unit of impedance is the $\Omega$ [6].

Henkin et al. [7] found that impedance values decreased significantly after the first stimulation (fitting) and that values remain stable 1 month after fitting.

However, Wolf-Magele et al. [8], studied 45 patients who received a CI; 40 patients were examined 2 weeks after the surgery. No statistically significant differences were found between the intraoperative, the 2-, and 6week postoperative impedance measurements for each channel, and it was concluded that earlier activation can be recommended.

The ECAP threshold is defined as the mean of the lowest positive response and the highest negative response measurements [9].

A typical ECAP pattern consists of two waveforms: a negative wave with a latency of $0.2-0.4 \mathrm{~ms}$ named (N1) and a positive peak or wave with a latency of $0.6-0.8 \mathrm{~ms}$ called (P1) [10].

Close to the compound action potentials in electrocochleography, the ECAPs are the sum of multiple neuron spikes, reflecting the neural synchronization after electrical stimulation, and can be obtained by a bidirectional stimulation-recording system through the implanted multichannel electrodes [11].

The ECAP test software from MED-EL was generated as auditory nerve response telemetry (ART), using the alternating polarity method for artifact rejection, and received approval from the FDA in 2007 [11].

Measurements of ECAP thresholds intraoperatively and postoperatively in the previous studies indicated that significant changes between the intraoperative and postoperative measures may or may not occur.

Lai et al. [12] and Tanamati et al. [13] reported no significant difference in ECAP thresholds in the first 12 months of the use of implants; they reported data obtained from a larger group of adult and pediatric CI users.

However, Gordon et al. [14] reported that ECAP thresholds measured at first stimulation were significantly decreased compared with those measured intraoperatively, with no significant difference in ECAP thresholds at later time points. Their data were collected from a group of children, and the same findings were reported by Telmesani et al. [15] in a group of 25 children.

\section{Rationale}

To date, few researches have compared the ECAP thresholds and impedance of the electrodes recorded at surgery, and their consistency during the postoperative period in patients receiving MED-EL cochlear implantable devices. Therefore, the aim of this study was to monitor ECAP Thresholds and the electrode impedance determined in the operation room, at initial fitting, and two time points after the initial stimulation a month apart to determine whether there is a significant difference in the values with time or not.

\section{Patients and methods}

Data were collected from 15 deaf children who were implanted unilaterally with a MED-EL Sonata Implant System with an Opus 2 speech processor.

All patients received implantation after they fulfilled the Alexandria main hospital criteria for receiving CIs. All children had been using hearing aids for a minimum time period of 3 months before undergoing implantation and had received speech rehabilitation.

Our study group included seven males and eight females and their ages ranged from 2 to 6 years (the mean age at the time of implantation was $3.42 \pm 1.17$ years). Two of 
these patients received an implant in the left ear, whereas the other patients received a CI in the right ear.

\section{Methods}

The 'activation' of the CI electrodes was performed 1 month after the surgery (to insure complete wound healing), which was the first activation time. Thus sending an electrical signal through the auditory nerve passing within the 12 intracochlear electrodes, and then the follow-up measurements were continued.

The electrode impedance and auditory response telemetry (ART) telemetry recordings were collected using MED-EL Maestro system software 2014, 6.0.1 version (Insbruke, Austria), to collect intraoperative and postoperative measurement values.

Connector cables between the external antenna and the max programming interface that was connected to a computer were used for bidirectional information recording of electrode impedance telemetry and ART (ECAP).

\section{Impedance measurement}

In this study, electrode impedance was measured intraoperatively. The results were accepted when the electrode impedance was less than $15 \mathrm{k} \Omega$. If the electrode impedance exceeded $20 \mathrm{k} \Omega$, the results were discarded because this reflects an open circuit.

The measurement was repeated in the initial stimulation visit (fitting) at two time points 1 month apart.

The impedance for all electrode pairs (channels) was calculated after the end of the second phase of biphasic stimulation pulses. The pulses used were qualitatively identical to pulses used for the normal stimulation mode of the implant.

The impedance was measured in the common ground mode. Values were considered normal when they were between 0.2 and $20 \mathrm{k} \Omega$. Electrodes with electrical problems such as short circuits, 'short' $(<0.2 \mathrm{k} \Omega)$, and open circuits, 'open' ( $>20 \mathrm{k} \Omega$ ), were not selected.

\section{Electrically evoked compound action potential measurements}

During the intraoperative period, the ART was recorded in selected electrodes E1, E5, E8, E11, and E12 because of restricted operating durations, and continued to be recorded postoperatively; the participants were evaluated at the following three time points:

(1) One month after the operation.
(2) Two months after the operation.

(3) Three months after the operation.

The parameters for ECAP recording were a maximum pulse of $500 \mathrm{CU}$ and if no response was obtained, this was increases to $700 \mathrm{CU}$ and recorded again, a phase duration of $35 \mu$ s, and an amplitude value of 8 . The current level of the masking noise was fixed at $10 \mathrm{U}$ above the stimulation level.

Recorded ART values had to have an identifiable ART waveform response, even though it is known that responses are increasingly less robust in the electrical threshold.

\section{Statistical analysis [16]}

Data were fed to the computer and analyzed using the IBM SPSS software package version 20.0 (IBM Corp, Armonk, New York, USA) [17]. Qualitative data were described using number and percent. The Kolmogorov-Smirnov test was used to verify the normality of distribution. Quantitative data were described using range (minimum and maximum), mean, SD, and median. The significance of the results obtained was judged at the $5 \%$ level.

The used test was analysis of variance with repeated measures. For normally quantitative variables, to compare between two or more periods or stages, and post-hoc test (LSD) for pairwise comparisons.

\section{Results}

Data from 15 children implanted with a MED-EL CI were collected in this work; seven were males and eight were females.

The youngest patient was 2 years old and the oldest patient was 6 years old when surgery was performed. All of the children had received implantation unilaterally in the right ear, except two patients, who received an implant in the left ear with full insertion of a standard electrode array (Tables 1 and 2).

Figure 1 shows that intraoperative impedance was the lowest among all postoperative readings in all electrodes. The highest value was that measured 1 month after surgery, after which impedance values continued to decrease significantly, but not the intraoperative values.

In Fig. 2, no significant changes in ECAP threshold were observed in the intraoperative period, and 1, 2, and 3 months after surgery. Although basal electrodes 
Table 1 Demographic data

\begin{tabular}{lc}
\hline & $N(\%)$ \\
\hline Sex & \\
$\quad$ Male & $7(46.7)$ \\
Female & $8(53.3)$ \\
Age (years) & \\
$<5$ & $13(86.7)$ \\
$\geq 5$ & $2(13.3)$ \\
Minimum-maximum & $2.0-5.75$ \\
Mean \pm SD & $3.42 \pm 1.17$ \\
Median & 3.0 \\
\hline
\end{tabular}

Table 2 Distribution of the studied cases according to site of implants $(n=15)$

\begin{tabular}{lc}
\hline Sites of implant & $N(\%)$ \\
\hline Left & $2(13.3)$ \\
Right & $13(86.7)$ \\
\hline
\end{tabular}

Figure 1

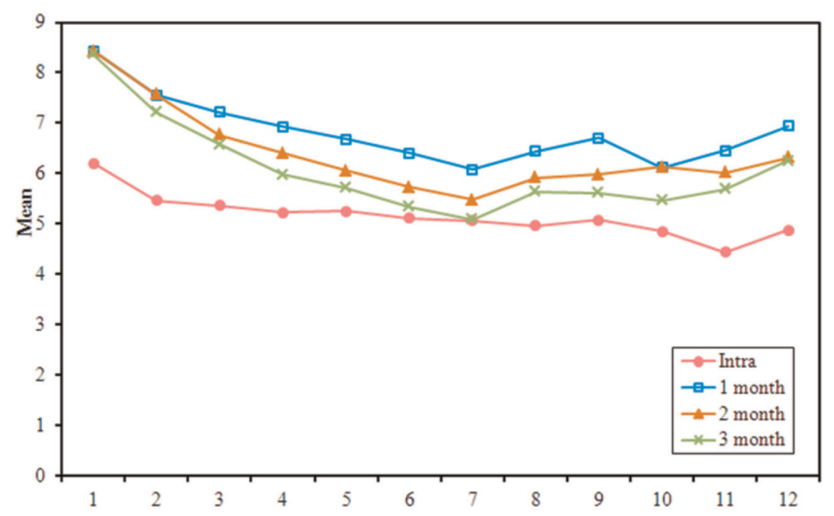

Comparison between impedance in different periods $(n=15)$.

(E11 and E12) showed different patterns in the form of no response intraoperatively and in the first stimulation period, ECAP thresholds were observed 2 months after surgery that were stable to the last reading, which was during the session in the third month.

\section{Discussion}

The aim of this work was to compare the electrode impedance and the ECAP threshold in patients with a unilateral $\mathrm{CI}$ over time.

The measurements were performed at four different intervals: at the time of the operation, during the stimulation session 1 month after surgery, 2 months after surgery, and 3 months after implantation. The measurements were carried out in 15 children ranging in age from 2 to 6 years at the time of implantation. An impedance value was obtained from all electrodes with no open or short circuits. The ECAP threshold was measured for electrodes $1,5,8,11$, and 12 , which represent apical, middle, and basal cochlear turns.
Figure 2

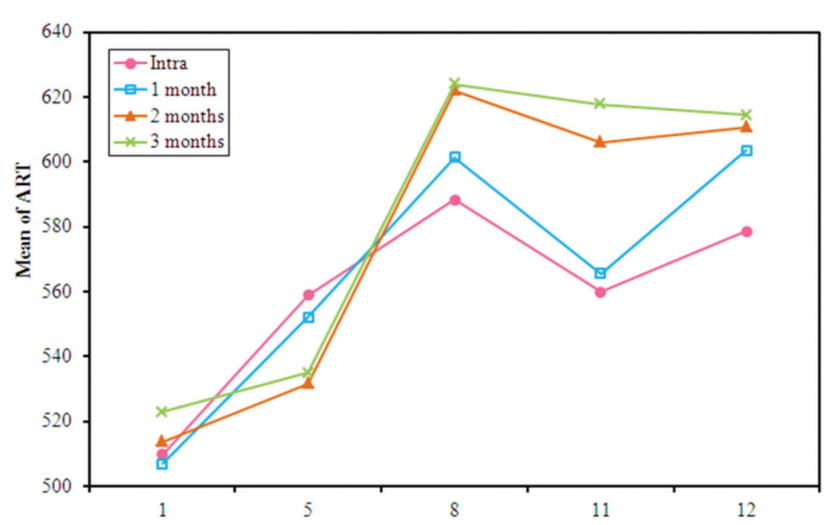

Comparison between different periods according to auditory nerve response telemetry (ART) $(n=15)$.

\section{Impedance analysis}

Our work indicated that the first-month postoperative electrode impedance showed a significant increase and started to decrease over the next 2 months in correlation to the intraoperative values. This increase in values could be attributed to the physiological changes and fibrosis that occur within the cochlea after electrode insertion. These findings were in agreement with studies carried out comparing intraoperative and postoperative impedances by Manolache et al. [4] and Sainz et al. [5], who reported an increase in the postoperative measured impedance compared with intraoperative values, and this was attributed to the physiological changes and fibrosis because of surgical trauma [9].

\section{Electrically evoked compound action potential} threshold

When the ECAP threshold was measured in our study at the time of the operation and at 1,2, and 3 months postoperatively the results showed insignificant changes in values comparing both intrasurgical and postsurgical measures. However, the mean ECAP threshold remained stable in all measured intervals with a mild increase in the ECAP threshold of basal electrodes 11 and 12.

Studies carried out by Lai et al. [12] and Tanamati et al. [13] reported no significant differences in the ECAP Thresholds during the first 12 months using CIs, either in adults or in children, and this was in agreement with our results.

However, Hughes et al. [9] studied 35 children and 33 adults with CIs between November 1996 and August 1999, and on comparing the EAP threshold, they found different results. After the 1-2-month followup visits, children showed significant increases in EAP thresholds, whereas these same measures in adults remained relatively stable. 
Also, Brown et al. [18] studied the same parameters and their results showed that ECAP thresholds and growth functions changed very little over a 5-6-year observation period, concluding that long-term use of a $\mathrm{CI}$ is not likely to have a significant negative impact on the response of the peripheral part of the auditory system. However, pediatric CI receivers were found to have, on average, higher ECAP thresholds and steeper ECAP growth functions than postlingually deafened adults who received CIs.

\section{Conclusion}

This research was carried out to identify whether there is a difference in telemetry measurements (impedance and ECAP) in populations with profound sensorineural hearing loss (SNHL) implanted unilaterally with MED-EL CIs. Both measurements were performed intraoperatively, 1 month after implantation (fitting cession), and 2 and 3 months after surgery. The study group included 15 children who ranged in age between 2 and 6 years at the time of the operation.

Measurements were collected from all electrodes for impedance and from ECAP, it was only collected for electrodes $1,5,8,11$, and 12 , which represent the cochlear apical, middle, and basal turns.

Impedance measurements showed a significant increase 1 month after surgery compared with intraoperative measurements and decreased significantly during the subsequent time points up to 3 months after surgery.

There was no significant difference between the ECAP threshold obtained in the intraoperative period and 1 , 2 , and 3 months after surgery, although basal electrode 11 showed different patterns in the form of no response intraoperatively and in the first stimulation period, but started to show ECAP thresholds less than or equal to $700 \mathrm{CU} 2$ months after surgery that remained stable thereafter.

(1) Because of differences in the follow-up durations and the groups studied in the telemetry measurements, studies with larger CI populations and longer durations must be carried out.

(2) ECAP measurements have to be performed in all electrodes to identify whether they show significant changes.

(3) These measurements could be performed by comparing different CI models.

(4) Impedance measurements should be carried out at each follow-up time point till stabilization of values.
(5) The ECAP threshold obtained intraoperatively is valuable for interpreting map in children.

\section{Acknowledgements}

This research was supported by the University of Alexandria. The authors thank their colleagues who provided insight and expertise.

\section{Financial support and sponsorship Nil}

\section{Conflicts of interest}

There are no conflicts of interest.

\section{References}

1 Bierer JA, Faulkner KF. Identifying cochlear implant channels with poor electrode-neuron interface: partial tripolar, single-channel thresholds and psychophysical tuning curves. Ear Hear 2010; 31:247-258.

2 Zwolan TA. Implants hearing devices. In: Katz J, Chasin M, English K, Hood JL, Tiller LK, editors. Hand book of clinical audiology. 7th ed. UK: Wolters Kluwer 2015. pp. 819-820.

3 Brill S, Müller J, Hagen R, Möltner A, Brockmeier SJ, Stark T, et al. Site of cochlear stimulation and its effect on electrically evoked compound action potentials using the MED-EL standard electrode array. Biomed Eng Online 2009; 8:40.

4 Manolache $O$. Electrical impedance variation values in patient with cochlear implant, Romanian. J Oral Rehabil 2012; 4:22-28.

5 Sainz M, Roldan C, de la Torre A, Gonzalez M, Ruiz J. Transitory alterations of the electrode impedances in cochlear implants associated to middle and inner ear diseases. Int Congr Ser 2003; 1240:407-410.

6 Dorman MF, Smith LM, Dankowski K, McCandless G, Parkin JL. Long-term measures of electrode impedance and auditory thresholds for the Ineraid cochlear implant. J Speech Hear Res 1992; 35:1126-1130.

7 Henkin Y, Kaplan-Neeman R, Muchnik C, Kronenberg J, Hildesheimer M. Changes over time in electrical stimulation levels and electrode impedance values in children using the Nucleus $24 \mathrm{M}$ cochlear implant. Int J Pediatr Otorhinolaryngol 2003; 67:873-880.

8 Wolf-Magele A, Schnabl J, Edlinger S, Pok SM, Schoerg P, Sprinzl GM. Postoperative changes in telemetry measurements after cochlear implantation and its impact on early activation, Clin Otolaryngol 2015; 40:527-534.

9 Hughes ML, Vander Werff KR, Brown CJ, Abbas PJ, Kelsay DM, Teagle $\mathrm{HF}$, et al. A longitudinal study of electrode impedance, the electrically evoked compound action potential, and behavioral measures in nucleus 24 cochlear implant users. Ear Hear 2001; 22:471-486.

10 Abbas PJ, Brown CJ, Shallop JK, Firszt JB, Hughes ML, Hong SH, et al. Summary of results using the nucleus Cl24M implant to record the electrically evoked compound action potential. Ear Hear 1999; 20:45-59.

11 Fei J, Ke L, Shi-Ming Y. Clinical application of electrically evoked compound action potentials. J Otol 2014; 9:117-121.

12 Lai WK, Aksit M, Akdas F, Dillier N. Longitudinal behavior of neural response telemetry (NRT) data and clinical implications. Int J Audiol 2004; 43:252-263.

13 Tanamati LF, Bevilacqua MC, Costa OA. Longitudinal study of the ECAP measured in children with cochlear implants. Braz J Otorhinolaryngol 2009; 75:90-96.

14 Gordon K, Papsin B, Harrison R. Toward a battery of behavioral and objective measures to achieve optimal cochlear implant stimulation levels in children. Ear Hear 2004; 25:447-463.

15 Telmesani LM, Said NM. Electrically evoked compound action potential (ECAP) in cochlear implant children: changes In auditory nerve response in first year of cochlear implant use. Int J Pediatr Otorhinolaryngol 2016; 82:28-33.

16 Kotz S, Balakrishnan N, Read CB, Vidakovic B. Encyclopedia of statistical sciences. 2nd ed. Hoboken, NJ: Wiley Interscience 2006.

17 Kirkpatrick LA, Feeney BC. A simple guide to IBM SPSS statistics for version 20.0, Student ed. Belmont, CA: Wadsworth, Cengage Learning 2013.

18 Brown CJ, Abbas PJ, Etlert CP, O'Brient S, Oleson JJ. Effects of long term use of a cochlear implant on the electrically evoked compound action potential. J Am Acad Audiol 2010; 21:5-15. 